Published in final edited form as:

Acad Radiol. 2018 December ; 25(12): 1588-1594. doi:10.1016/j.acra.2018.02.027.

\title{
Does enhancement or perfusion on pre-procedure CT predict outcomes after embolization of hepatocellular carcinoma?
}

\author{
Alessandra Borgheresi, MD, \\ Radiodiagnostic Section, Department of Clinical Physiopathology, University of Florence, Azienda \\ Ospedaliero-Universitaria Careggi, Largo Brambilla 3, Florence 50134, Italy \\ Adrian Gonzalez-Aguirre, MD, \\ Interventional Radiology Service, Department of Radiology, Memorial Sloan Kettering Cancer \\ Center, 1275 York Ave., New York, NY 10065 \\ Karen T Brown, MD, \\ Interventional Radiology Service, Department of Radiology, Memorial Sloan Kettering Cancer \\ Center, 1275 York Ave., New York, NY 10065 \\ George I Getrajdman, MD, \\ Interventional Radiology Service, Department of Radiology, Memorial Sloan Kettering Cancer \\ Center, 1275 York Ave., New York, NY 10065 \\ Joseph P Erinjeri, MD, PhD, \\ Interventional Radiology Service, Department of Radiology, Memorial Sloan Kettering Cancer \\ Center, 1275 York Ave., New York, NY 10065
}

Anne Covey, MD, Interventional Radiology Service, Department of Radiology, Memorial Sloan Kettering Cancer Center, 1275 York Ave., New York, NY 10065

Hooman Yarmohammadi, MD, Interventional Radiology Service, Department of Radiology, Memorial Sloan Kettering Cancer Center, 1275 York Ave., New York, NY 10065

Correspondence to: F. Edward Boas.

Author contributions:

Study design: AB, FEB, AG, GIG

Data collection: $\mathrm{AB}$

Data analysis: AB, FEB, GIG

Drafting manuscript: AB, FEB

Revising manuscript: AB, AG, KTB, GIG, JPE, AC, HY, EZ, CS, FEB

Final approval of manuscript: FEB

Disclosures

FEB is a co-founder of Claripacs, LLC. He received research supplies from Bayer (sorafenib). He is an investor in Labdoor, Qventus, CloudMedx, and Notable Labs.

CTS is a consultant for Sirtex, Neuwave, Johnson \& Johnson, and GE. He currently receives research support from TheraSphere/ Biocompatibles.

Publisher's Disclaimer: This is a PDF file of an unedited manuscript that has been accepted for publication. As a service to our customers we are providing this early version of the manuscript. The manuscript will undergo copyediting, typesetting, and review of the resulting proof before it is published in its final citable form. Please note that during the production process errors may be discovered which could affect the content, and all legal disclaimers that apply to the journal pertain. 
Etay Ziv, MD, PhD, Interventional Radiology Service, Department of Radiology, Memorial Sloan Kettering Cancer Center, 1275 York Ave., New York, NY 10065

Constantinos T. Sofocleous, MD, PhD, and Interventional Radiology Service, Department of Radiology, Memorial Sloan Kettering Cancer Center, 1275 York Ave., New York, NY 10065

F. Edward Boas, MD, PhD Interventional Radiology Service, Department of Radiology, Memorial Sloan Kettering Cancer Center, 1275 York Ave., New York, NY 10065

\section{Abstract}

Purpose-To evaluate whether quantitative enhancement or perfusion measurements on preprocedure triphasic CT can be used to predict response or overall survival after embolization of hepatocellular carcinoma (HCC).

Materials and Methods-The Institutional Review Board approved this retrospective review of 63 patients with HCC treated with particle embolization between March 2009 and December 2014. Quantitative enhancement and perfusion measurements were performed on the target tumor and the background liver on the triphasic CT obtained prior to treatment. Microvascular invasion (MVI) and degree of differentiation were determined from a core biopsy specimen. Quantitative enhancement and perfusion values were then correlated with pathology (two-tailed $t$-test), response to embolization on mRECIST (two-tailed $t$-test), and overall survival after embolization (Cox proportional hazards model).

Results-Arterial enhancement did not predict immediate response or overall survival after embolization. The degree of differentiation or presence of microvascular invasion also did not predict immediate response or overall survival after embolization. However, high hepatic artery coefficient (HAC) or low portal vein coefficient (PVC), both in the tumor $(p=0.011$ and $p=0.004)$ and in the background liver $(p=0.015$ and $p=0.009)$, were associated with worse survival. HAC, both in the tumor $(p=0.025)$ and the background liver $(p=0.013)$, were independent predictors of survival in a multivariate model including Child Pugh and BCLC.

Conclusion-Tumor and liver perfusion parameters estimated from pre-procedure triphasic CT were predictive of survival after embolization. Arterial phase enhancement and histology (degree of differentiation or MVI) did not predict immediate response or overall survival after particle embolization.

\section{Keywords}

hepatocellular carcinoma; cirrhosis; perfusion; blood supply; hypovascular

\section{Introduction}

Treatment options for unresectable and unablatable hepatocellular carcinoma (HCC) include transarterial embolization (TAE), transarterial chemoembolization (TACE) either using lipiodol or drug-eluting beads, radioembolization, and sorafenib. Recent randomized trials 
have shown no difference in overall survival for these treatment options, although there are differences in response rates and quality of life (1-3).

Regardless of the treatment, there are a subset of patients who do not respond $(1,3)$.

Although overall survival after the various different treatment options for unresectable HCC appears to be similar, there might be subgroups that do better with one treatment compared to another (4). Predicting which patients will have poor outcomes with one treatment option could potentially allow these patients to be directed to a different treatment option.

Intuitively, hypervascular tumors should have a better response to an arterially directed therapy, but the literature on this topic is mixed. Some studies showed that the degree of enhancement of HCC on arterial phase CT predicts response to TACE or TAE $(5,6)$, but other studies showed no correlation between enhancement and response $(7,8)$. For radioembolization of liver metastases, hypervascularity on arterial phase CT or MRI was not correlated with response or survival $(9,10)$.

Liver tumors that appear hypovascular on CT or MRI frequently appear to be hypervascular on catheter angiography (9). Tumor enhancement on catheter angiography is a better predictor of response to embolization than tumor enhancement on CT (8), suggesting that better noninvasive methods for determining tumor vascularity could be helpful.

We recently developed a non-invasive method for estimating the arterial and portal venous blood supply to liver lesions, based on quantitative measurements on standard pre-procedure triphasic CT scans (11). Using this method, we were able to quantitate the increased hepatic artery perfusion in hepatocellular carcinoma, and the decreased portal perfusion in cirrhotic livers (11). In addition, these perfusion parameters estimated from triphasic CT can be used to predict response of colorectal liver metastases to radioembolization (10). However, it is unknown whether perfusion parameters predict outcomes after embolization of HCC.

The purpose of this study is to examine whether quantitative perfusion or enhancement measurements on routine pre-procedure multi-phase $\mathrm{CT}$ can be used to predict the response or overall survival after embolization of HCC. In particular, we examined arterial phase enhancement, hepatic artery coefficient (HAC), portal vein coefficient (PVC), and arterial enhancement fraction (AEF) of the HCC and the background liver. HAC is an estimate of the hepatic artery blood supply; PVC is an estimate of the portal vein blood supply; and AEF is related to the arterial blood supply as a fraction of total blood supply (10).

\section{Materials and methods}

\section{Patient selection}

The Institutional Review Board approved this HIPAA-compliant retrospective study. Inclusion criteria were: patients with $\mathrm{HCC}$ with no prior locoregional therapy, who received TAE or DEB-TACE between March 2009 and December 2014, had at least one measurable target tumor according to mRECIST guidelines (12), and had triphasic CT studies performed within 2 months before and at 3 months after the procedure. Patients with main portal vein 
thrombus or infiltrative HCC were excluded. HCC was diagnosed based on pathology (if available) or imaging.

The final study population included 63 patients ( 10 women and 53 men), with median age of 66 years (range, 25-86 years). A retrospective review of the medical records was performed to determine the Child-Pugh (CP) score, Barcelona Clinic Liver Cancer (BCLC) stage, etiology of liver disease, and pathology results (degree of differentiation and presence of microvascular invasion).

\section{Transarterial embolization}

Embolization of HCC was performed by a board-certified, fellowship-trained interventional radiologist, using a standard protocol $(3,13)$. Embolization was performed as selectively as possible, starting with $40-120$ or $100-300 \mu \mathrm{m}$ spherical particles. For DEB-TACE, doxorubicin $150 \mathrm{mg}$ was loaded onto 100-300 $\mu \mathrm{m}$ LC Beads. For both TAE and DEBTACE, embolization was performed to stasis, which sometimes required use of larger spherical particles, or irregularly shaped PVA foam particles (Supplemental Table 2).

\section{Imaging protocol}

Triphasic liver CT was performed according to our standard clinical protocol. After obtaining a non-contrast CT, $150 \mathrm{ml}$ of iohexol $300 \mathrm{mg} \mathrm{I} / \mathrm{ml}$ (Omnipaque 300, GE Healthcare, Little Chalfont, United Kingdom) was power injected intravenously at $4 \mathrm{ml} / \mathrm{s}$. An arterial phase scan was obtained 9 seconds after the abdominal aorta at the level of the celiac artery reached 150 Hounsfield units (HU). This scan was typically obtained around 30 seconds after the start of contrast injection. A portal venous phase scan was obtained 40 seconds after the arterial phase scan. All scans were performed at a kVp of 120.

\section{Image evaluation}

For each patient, the largest measurable treated target tumor was selected. This target tumor was used to evaluate enhancement, perfusion, and mRECIST response. Elliptical regions of interest (ROI) were placed on the hepatic artery, portal vein, target tumor, and background liver on each of the three phases of the pre-treatment triphasic CT. The ROIs had the same size, shape, and anatomic location on each phase. The target tumor ROIs covered the entire tumor on a single slice, excluding large intratumoral vessels. For each ROI, the mean attenuation value in Hounsfield units (HU) was recorded. Arterial and portal venous phase enhancement (relative to the non-contrast phase), HAC, PVC, and AEF were then calculated for each target tumor and the background liver (Supplemental Table 1), as previously described (10).

Response to treatment was evaluated on triphasic CT obtained approximately 3 months after the procedure. Target tumor response was assessed according to mRECIST (12). Tumors with complete response (CR) were compared to tumors with partial response (PR), stable disease (SD), or progressive disease (PD). ROI measurements were performed by A.B. (5 years of experience in abdominal CT) on a clinical PACS workstation (Centricity PACS, GE Healthcare, Barrington, IL). Imaging response was independently evaluated by A.B. and 
F.E.B., and discrepancies were evaluated by A.G. $(5,8$, and 11 years of experience in abdominal CT, respectively).

\section{Statistical analysis}

Cohen's kappa coefficient was used to evaluate inter-rater agreement for mRECIST response. Differences in tumor enhancement and perfusion values were evaluated by using two-tailed $t$-tests. Differences in pathology (degree of differentiation or presence of microvascular invasion) were evaluated by using Fisher's exact test. Kaplan Meier survival curves were compared using a log rank test. Overall survival after initial embolization was predicted using univariate and multivariate Cox proportional hazards models. $P$ values less than 0.05 were considered significant. HAC was classified as high or low, using a cutoff value that was selected to minimize the $p$ value (14) for comparing the Kaplan Meier survival curves for patients above versus below the cutoff, using the log rank test.

\section{Results}

\section{Patient characteristics}

At time of embolization, most patients were Child Pugh A and BCLC B (Table 1).

The most common etiology of liver disease was HCV, and most patients had cirrhosis (based on CT findings or biopsy). Mean target tumor size at baseline was $5.4 \mathrm{~cm}$ (range 1-35). Core biopsy or liver resection pathology was available in 54 of 63 patients. Core biopsy was obtained in patients whose imaging was not diagnostic of HCC (11 patients), in patients without cirrhosis or hepatitis B (16 patients), to confirm metastatic disease (2 patients), as part of a biospecimen protocol (1 patient), per oncology recommendations (1 patient), or was obtained at an outside hospital (20 patients). Pathology showed that most tumors were well to moderately differentiated, and microvascular invasion (MVI) was seen in 9 of 54 patients (17\%). In 9 of 63 patients, pathology was not available; all of these patients were LI-RADS 5 based on CT.

\section{Predicting response to embolization}

After embolization, 31 (49\%) of target tumors had a complete response, 26 (41\%) had a partial response, 4 (6\%) patients had stable disease, and 2 patients (3\%) had progressive disease, using mRECIST criteria. Cohen's kappa coefficient was 0.70 for the mRECIST readers. Particle size did not predict complete response (Supplemental Table 3). Preprocedure perfusion parameters (HAC, $\mathrm{PVC}, \mathrm{AEF}$ ) and enhancement measurements (in the arterial and portal venous phases) did not predict complete response to embolization (Table 2, Figure 1). Pathology (degree of differentiation and MVI) also did not predict complete response to embolization.

\section{Predicting overall survival after embolization}

Median overall survival in the study population was 29 months (95\% CI, 22-45). The median follow-up time after embolization was 28 months and at the time of data collection, 36 patients were dead. Particle size did not predict overall survival (Supplemental Table 3, Supplemental Figure 1). We used a Cox proportional hazards model to try to predict survival 
based on patient characteristics and perfusion parameters (Table 3). As expected, BCLC C is associated with much worse survival than BCLC A or B. Child Pugh was not significantly predictive of survival in our patient population, which was almost exclusively Child Pugh A.

Pre-procedure tumor arterial enhancement did not predict overall survival after embolization (Table 3). However, high HAC or low PVC (both in the tumor and in the background liver) were associated with worse survival (Figure 3). HAC and PVC were negatively correlated $\left(r^{2}=0.73\right.$ and $p=3.4 \times 10^{-19}$ for the tumor; $r^{2}=0.95$ and $p=4.1 \times 10^{-42}$ for the liver). HAC in the tumor was not correlated with HAC in the background liver $\left(r^{2}=0.02, p=0.26\right)$ and PVC in the tumor was not correlated with PVC in the background liver $\left(r^{2}=0.05, p=0.076\right)$. A multivariate Cox regression analysis, including Child Pugh and BCLC, showed that HAC values (both in the tumor and the background liver) were independent predictors of survival (Table 3).

Pathology (MVI and degree of differentiation) did not predict overall survival after embolization (Figure 2).

\section{Predicting pathology from imaging}

HCCs with more arterial phase enhancement were more likely to be moderately to poorly differentiated (Table 4). However, enhancement and perfusion parameters were not correlated with presence of microvascular invasion $(p>0.05)$.

\section{Discussion}

Hypovascular and hypervascular HCC (based on pre-procedure arterial phase CT) have similar immediate response and overall survival after embolization. One possible explanation for this somewhat counterintuitive finding is that hypervascular HCC is more likely to be moderately to poorly differentiated. Thus, increased arterial flow of embolic particles to hypervascular HCC might be counterbalanced by the more aggressive pathology.

The data also show that pathology (degree of differentiation and microvascular invasion) does not predict response or survival after embolization of HCC. This agrees with prior studies (15). In contrast, after resection or liver transplantation, patients with poorly differentiated HCC or microvascular invasion had worse outcomes (16-18). Thus, pathology appears to predict outcomes after surgery, but not after embolization. This discrepancy might be explained by the increased arterial vascularity of more aggressive pathologies, which might increase flow of embolic particles to the tumor.

In other words, there are two separate factors that work in opposite directions after embolization of HCC. 1. Hypervascular HCC is more likely to be moderately to poorly differentiated, and thus have worse outcomes. 2. Hypervascular HCC probably has better preferential flow of embolic particles to tumor, and thus have better outcomes after embolization. These two opposing factors appear to cancel each other out in our patient population, and thus, there was no difference in outcomes after embolization of hypovascular versus hypervascular HCC (when vascularity is determined based on arterial phase enhancement). 
The major new result in this paper is that perfusion parameters (high HAC and low PVC) in both the HCC and the background liver were independent predictors of worse survival, after accounting for BCLC stage and Child Pugh score. The liver and tumor perfusion parameters were uncorrelated, and thus provided independent information on survival. However, perfusion parameters did not predict response to embolization.

The perfusion data show that HCC with more hepatic artery supply (HAC), or less portal vein supply (PVC), had worse survival after embolization. The switch from portal vein to hepatic artery supply is a key step in HCC progression $(19,20)$. However, pathology (degree of differentiation or microvascular invasion) did not predict survival. This suggests that perfusion parameters might be more accurate at detecting aggressive tumors than degree of differentiation or microvascular invasion on a core biopsy. A core biopsy only samples a small fraction of a heterogeneous tumor, while the perfusion parameters sample most of the tumor volume on an axial slice. Furthermore, biopsies are typically not obtained when imaging is diagnostic of HCC, so perfusion information might be especially useful for those patients.

The perfusion data also show that livers with more hepatic artery supply (HAC), or less portal vein supply (PVC), had worse survival after embolization. Cirrhosis is associated with decreased portal flow, which can be detected as decreased PVC (11). In our data, liver HAC and PVC were negatively correlated, consistent with the well-known hepatic arterial buffer response, whereby the liver compensates for reduced portal flow by increasing hepatic arterial flow (21). The predictive value of perfusion parameters remained significant in the multivariate model, which includes the numeric Child-Pugh score. Thus, the perfusion parameters appear to detect early cirrhotic changes that are not reflected in the Child-Pugh score, but that are still important for predicting survival after embolization.

These results highlight the fact that underlying liver dysfunction is an important determinant of outcomes, and that a normal Child Pugh score does not mean that the liver is normal. The perfusion parameters appear to detect abnormal liver function that is not reflected in the Child Pugh score. This could be important information for deciding on the right balance between aggressively treating the tumor versus trying to preserve liver function, as an HCC patient could die either from HCC disease progression, or liver failure related to cirrhosis and treatment.

There are several limitations to this study. First, this is a retrospective single-institution study in a small group of patients, and the results should be validated at other institutions. Second, only a single tumor was analyzed in each patient. Third, enhancement measurements were performed on single axial slices, rather than examining the entire tumor volume. Fourth, infiltrative HCCs were excluded, as they are non-measurable tumors according to mRECIST.

In conclusion, arterial phase enhancement of HCC does not predict outcomes after embolization, but tumor and liver perfusion parameters estimated from pre-embolization triphasic CT can predict overall survival. The perfusion parameters were better predictors of survival than the Child Pugh score or histology of the HCC. These perfusion parameters can be calculated from Hounsfield unit measurements obtained using a clinical PACS 
workstation, without requiring any special software or scanning protocols. Future work should examine whether this can be used to guide the optimal treatment strategy for each patient, and whether the Child-Pugh score could be modified to include early cirrhotic changes detected using perfusion parameters.

\section{Supplementary Material}

Refer to Web version on PubMed Central for supplementary material.

\section{Acknowledgments}

This research was funded in part through an NIH/NCI Cancer Center Support Grant (P30 CA008748).

\section{References}

1. Salem R, Gordon AC, Mouli S, et al. Y90 Radioembolization Significantly Prolongs Time to Progression Compared With Chemoembolization in Patients With Hepatocellular Carcinoma. Gastroenterology. 2016; 151(6):1155-63e2. [PubMed: 27575820]

2. Vilgrain V, Bouattour M, Sibert A, et al. SARAH: A randomised controlled trial comparing efficacy and safety of selective internal radiation therapy (with yttrium-90 microspheres) and sorafenib in patients with locally advanced hepatocellular carcinoma. Journal of Hepatology. 2017; 66(1, Supplement):S85-6.

3. Brown KT, Do RK, Gonen M, et al. Randomized Trial of Hepatic Artery Embolization for Hepatocellular Carcinoma Using Doxorubicin-Eluting Microspheres Compared With Embolization With Microspheres Alone. J Clin Oncol. 2016; 34(17):2046-53. [PubMed: 26834067]

4. Biederman DM, Titano JJ, Tabori NE, et al. Outcomes of Radioembolization in the Treatment of Hepatocellular Carcinoma with Portal Vein Invasion: Resin versus Glass Microspheres. J Vasc Interv Radiol. 2016; 27(6):812-21e2. [PubMed: 27062356]

5. Katyal S, Oliver JH, Peterson MS, Chang PJ, Baron RL, Carr BI. Prognostic significance of arterial phase CT for prediction of response to transcatheter arterial chemoembolization in unresectable hepatocellular carcinoma: a retrospective analysis. AJR Am J Roentgenol. 2000; 175(6):1665-72. [PubMed: 11090401]

6. Hu HT, Kim JH, Lee LS, et al. Chemoembolization for hepatocellular carcinoma: multivariate analysis of predicting factors for tumor response and survival in a 362-patient cohort. J Vasc Interv Radiol. 2011; 22(7):917-23. [PubMed: 21571545]

7. Artinyan A, Nelson R, Soriano P, et al. Treatment response to transcatheter arterial embolization and chemoembolization in primary and metastatic tumors of the liver. HPB (Oxford). 2008; 10(6):396404. [PubMed: 19088924]

8. Kwan SW, Fidelman N, Ma E, Kerlan RK Jr, Yao FY. Imaging predictors of the response to transarterial chemoembolization in patients with hepatocellular carcinoma: a radiologicalpathological correlation. Liver Transpl. 2012; 18(6):727-36. [PubMed: 22344899]

9. Sato KT, Omary RA, Takehana C, et al. The role of tumor vascularity in predicting survival after yttrium-90 radioembolization for liver metastases. J Vasc Interv Radiol. 2009; 20(12):1564-9. [PubMed: 19846320]

10. Boas FE, Brody LA, Erinjeri JP, et al. Quantitative Measurements of Enhancement on Preprocedure Triphasic CT Can Predict Response of Colorectal Liver Metastases to Radioembolization. AJR Am J Roentgenol. 2016; 207(3):671-5. [PubMed: 27248430]

11. Boas FE, Kamaya A, Do B, et al. Classification of hypervascular liver lesions based on hepatic artery and portal vein blood supply coefficients calculated from triphasic CT scans. Journal of digital imaging. 2015; 28:213-23. [PubMed: 25183580]

12. Lencioni R, Llovet JM. Modified RECIST (mRECIST) assessment for hepatocellular carcinoma. Semin Liver Dis. 2010; 30(1):52-60. [PubMed: 20175033] 
13. Brown KT. Bland embolization for hepatic malignancies. In: Mauro MA, Murphy KPJ, Thomson KR, Venbrux AC, Morgan RA, editorsImage-Guided Interventions. Elsevier; 2014. 467-75.

14. Budczies J, Klauschen F, Sinn BV, et al. Cutoff Finder: a comprehensive and straightforward Web application enabling rapid biomarker cutoff optimization. PloS one. 2012; 7(12):e51862. [PubMed: 23251644]

15. Herber S, Biesterfeld S, Franz U, et al. Correlation of multislice CT and histomorphology in HCC following TACE: predictors of outcome. Cardiovasc Intervent Radiol. 2008; 31(4):768-77. [PubMed: 18196335]

16. Lim KC, Chow PK, Allen JC, et al. Microvascular invasion is a better predictor of tumor recurrence and overall survival following surgical resection for hepatocellular carcinoma compared to the Milan criteria. Annals of surgery. 2011; 254(1):108-13. [PubMed: 21527845]

17. Jonas S, Bechstein WO, Steinmuller T, et al. Vascular invasion and histopathologic grading determine outcome after liver transplantation for hepatocellular carcinoma in cirrhosis. Hepatology. 2001; 33(5):1080-6. [PubMed: 11343235]

18. Lee EC, Kim SH, Park H, Lee SD, Lee SA, Park SJ. Survival analysis after liver resection for hepatocellular carcinoma: A consecutive cohort of 1002 patients. J Gastroenterol Hepatol. 2017; 32(5):1055-63. [PubMed: 27797420]

19. Choi JY, Lee JM, Sirlin CB. CT and MR imaging diagnosis and staging of hepatocellular carcinoma: part I. Development, growth, and spread: key pathologic and imaging aspects. Radiology. 2014; 272(3):635-54. [PubMed: 25153274]

20. Kitao A, Zen Y, Matsui O, Gabata T, Nakanuma Y. Hepatocarcinogenesis: multistep changes of drainage vessels at CT during arterial portography and hepatic arteriography--radiologicpathologic correlation. Radiology. 2009; 252(2):605-14. [PubMed: 19703890]

21. Gulberg V, Haag K, Rossle M, Gerbes AL. Hepatic arterial buffer response in patients with advanced cirrhosis. Hepatology. 2002; 35(3):630-4. [PubMed: 11870377] 
A.

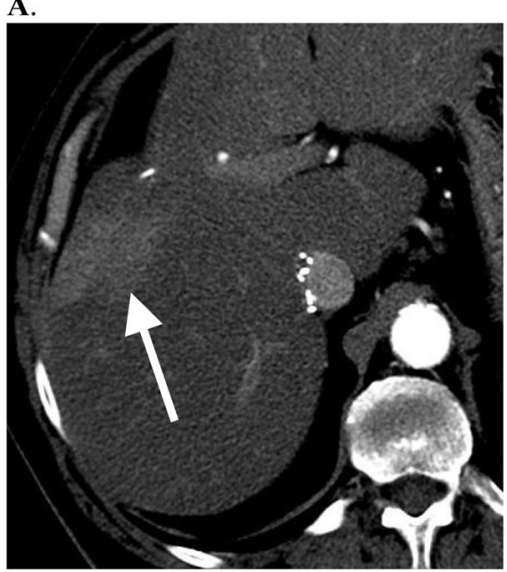

C.

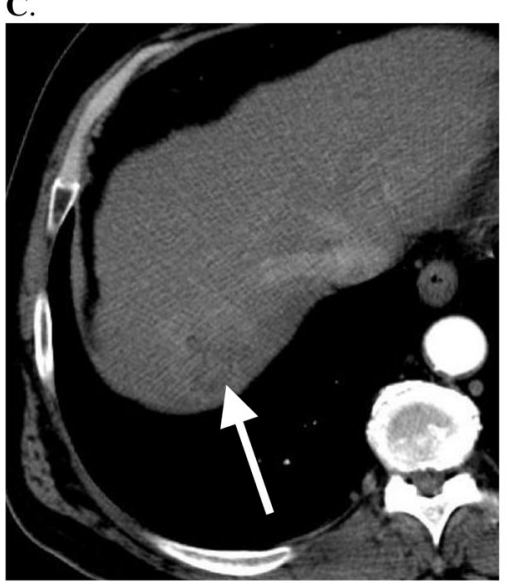

B.

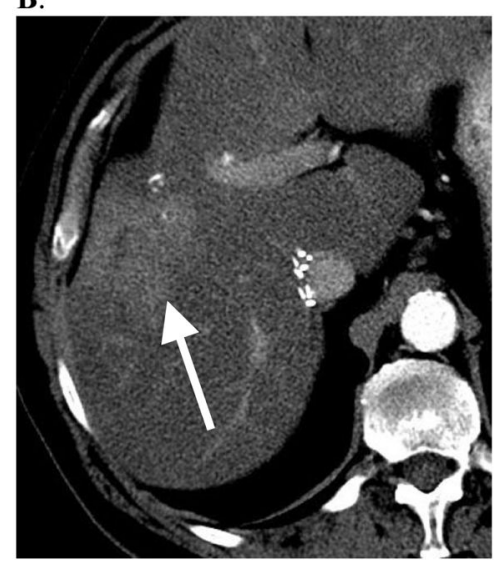

D.

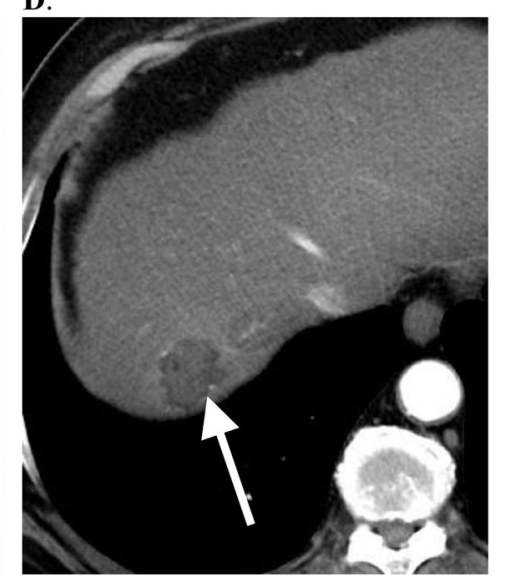

Figure 1.

Tumor arterial enhancement did not predict response to embolization or overall survival. Examples show that hypervascular HCC can fail to respond to embolization, and hypovascular HCC can respond to embolization. A. 67-year-old male with HCC, which appears hypervascular on arterial phase CT. B. This tumor progressed after embolization. C. 62-year-old male with HCC, which appears hypovascular on arterial phase CT. D. This tumor appears necrotic after embolization. 
A.

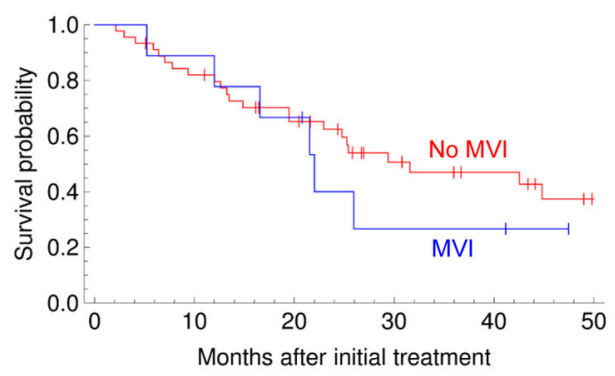

B.

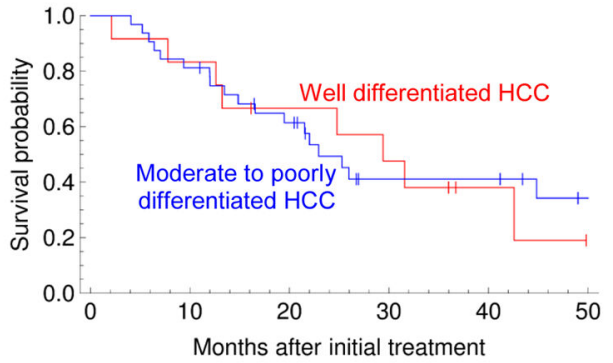

Figure 2.

Histology (microvascular invasion and degree of differentiation from a core biopsy) did not predict overall survival after embolization. A. Red: no microvascular invasion, Blue: microvascular invasion $(p=0.45)$. B. Red: well differentiated HCC, Blue: moderate or poorly differentiated HCC $(p=0.87)$. 
A.

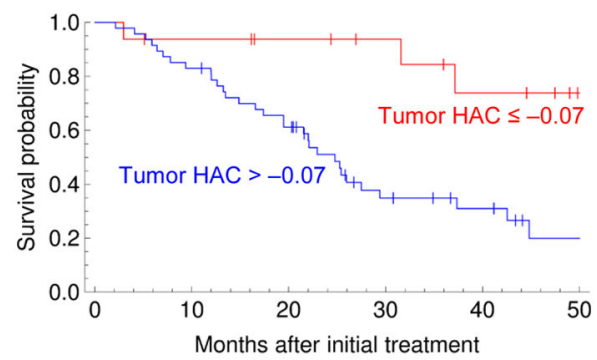

B.

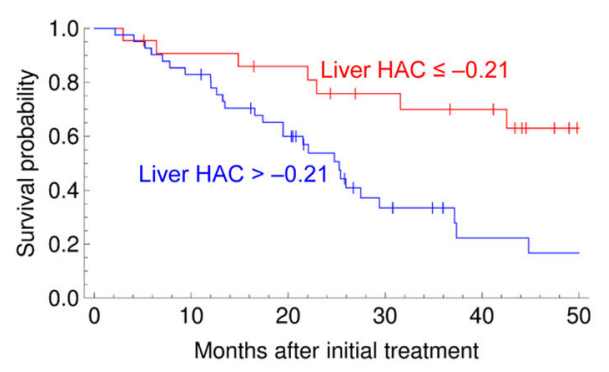

Figure 3.

Perfusion parameters in both the HCC and the background liver are independent predictors of overall survival (see Table 3 for $p$ values). A. Red: tumor HAC $\leq-0.07$, Blue: tumor HAC $>-0.07$. B. Red: liver HAC $\leq-0.21$, Blue: liver HAC $>-0.21$. 


\section{Table 1}

Patient characteristics. HBV: Hepatitis B virus; HCV: Hepatitis C virus; NASH: non-alcoholic liver steatohepatitis; MVI: microvascular invasion.

\begin{tabular}{|c|c|c|}
\hline & & $\mathbf{N}(\%)$ \\
\hline \multirow[t]{3}{*}{ Child-Pugh class } & $\mathrm{A}$ & $58(92 \%)$ \\
\hline & B & $5(8 \%)$ \\
\hline & $\mathrm{C}$ & $0(0 \%)$ \\
\hline \multirow[t]{4}{*}{ BCLC stage } & 0 & $1(1 \%)$ \\
\hline & $\mathrm{A}$ & $18(29 \%)$ \\
\hline & $\mathrm{B}$ & $34(54 \%)$ \\
\hline & $\mathrm{C}$ & $10(16 \%)$ \\
\hline \multirow[t]{6}{*}{ Etiology liver disease } & HBV & $10(16 \%)$ \\
\hline & $\mathrm{HCV}$ & $18(29 \%)$ \\
\hline & Alcoholism & $6(10 \%)$ \\
\hline & NASH & $5(8 \%)$ \\
\hline & Other & $18(29 \%)$ \\
\hline & Multiple & $5(8 \%)$ \\
\hline \multirow[t]{2}{*}{ Cirrhosis } & Yes & $37(59 \%)$ \\
\hline & No & $26(41 \%)$ \\
\hline \multirow[t]{7}{*}{ Pathology (degree of differentiation) } & Well-differentiated & $12(19 \%)$ \\
\hline & Moderately-differentiated & $26(41 \%)$ \\
\hline & Poorly-differentiated & $6(10 \%)$ \\
\hline & Undifferentiated & $0(0 \%)$ \\
\hline & Fibrolamellar & $2(3 \%)$ \\
\hline & Not specified & $8(13 \%)$ \\
\hline & Core biopsy not performed & $9(14 \%)$ \\
\hline \multirow[t]{3}{*}{ Pathology (MVI) } & Yes & $9(14 \%)$ \\
\hline & No & $45(71 \%)$ \\
\hline & Core biopsy not performed & $9(14 \%)$ \\
\hline
\end{tabular}




\section{Table 2}

HCC enhancement, perfusion parameters, and pathology did not predict complete response after embolization.

\begin{tabular}{llll}
\hline & $\begin{array}{l}\text { CR } \\
\text { Av. } \pm \text { SD }\end{array}$ & $\begin{array}{l}\text { Non-CR } \\
\text { Av. } \pm \text { SD }\end{array}$ & $\boldsymbol{p}$ value \\
\hline HAC & $0.03 \pm 0.22$ & $0.08 \pm 0.29$ & 0.45 \\
PVC & $0.44 \pm 0.27$ & $0.31 \pm 0.41$ & 0.14 \\
AEF & $0.74 \pm 0.25$ & $0.94 \pm 0.83$ & 0.21 \\
Arterial phase enhancement $($ HU) & $44 \pm 17$ & $45 \pm 36$ & 0.86 \\
Portal venous phase enhancement $(\mathbf{H U})$ & $60 \pm 15$ & $53 \pm 20$ & 0.13 \\
Moderate to poorly differentiated & $12 / 16$ & $20 / 28$ & 1.0 \\
MVI & $5 / 25$ & $4 / 29$ & 0.72 \\
\hline
\end{tabular}


Table 3

Predictors of overall survival after embolization of HCC (Cox proportional hazards model).

\begin{tabular}{llll}
\hline & & $\begin{array}{l}\text { Univariate model } \\
\text { Relative risk }(\boldsymbol{p} \text { value })\end{array}$ & $\begin{array}{l}\text { Multivariate model } \\
\text { Relative risk }(\boldsymbol{p} \text { value })\end{array}$ \\
\hline Tumor & HAC & $3.85\left(0.011^{*}\right)$ & $4.38\left(0.025^{*}\right)$ \\
& PVC & $0.32\left(0.004^{*}\right)$ & \\
& AEF & $1.39(0.115)$ & \\
& Arterial enhancement $(\mathrm{HU})$ & $1(0.73)$ & \\
& PV enhancement $(\mathrm{HU})$ & $0.99(0.28)$ & $1.33\left(0.0133^{*}\right)$ \\
\hline Liver & HAC & $1.27\left(0.015^{*}\right)$ & $1.08(0.684)$ \\
\hline Child Pugh & PVC & $0.75\left(0.009^{*}\right)$ & $1(\mathrm{n} / \mathrm{a})$ \\
\hline BCLC & 0 or A & $1.04(0.83)$ & $0.86(0.725)$ \\
& B & $0.69(0.34)$ & $6.31(0.00016 *)$ \\
\hline * & C & $0.55(0.084)$ &
\end{tabular}




\section{Table 4}

Higher arterial or portal venous phase enhancement predicts moderate to poorly differentiated HCC.

\begin{tabular}{llll}
\hline & $\begin{array}{l}\text { Well-differentiated } \\
\text { Av. } \pm \text { SD }\end{array}$ & $\begin{array}{l}\text { Moderately to poorly differentiated } \\
\text { Av. } \pm \text { SD }\end{array}$ & $\boldsymbol{p}$ value \\
\hline Arterial phase enhancement $($ HU) & $29 \pm 16$ & $52 \pm 52$ & $0.004^{*}$ \\
PV enhancement $($ HU) & $43 \pm 19$ & $58 \pm 58$ & $0.028^{*}$ \\
HAC & $0.02 \pm 0.19$ & $0.10 \pm 0.10$ & 0.345 \\
PVC & $0.27 \pm 0.34$ & $0.37 \pm 0.37$ & 0.415 \\
AEF & $0.94 \pm 0.99$ & $0.91 \pm 0.91$ & 0.922 \\
\hline
\end{tabular}

statistically significant difference. 